\title{
Influence of MTHFR C677T and A1298C polymorphisms on the survival of pediatric patients with non-Hodgkin lymphoma
}

\author{
Xiaoqin Zhu ${ }^{1}$, wei li ${ }^{2}$, Jia Zhu ${ }^{3}$, Huimou Chen ${ }^{1}$, Jinqiu Guan ${ }^{1}$, Dalei Zhou ${ }^{1}$, Zijun Zhen ${ }^{1}$, \\ Feifei Sun ${ }^{1}$, Junting Huang ${ }^{3}$, Juan Wang ${ }^{3}$, Xiaofei Sun ${ }^{4}$, Zhang Yizhuo ${ }^{1}$, and Su-Ying Lu ${ }^{1}$ \\ ${ }^{1}$ Sun Yat-sen University Cancer Center \\ ${ }^{2}$ Guangzhou Women and Children's Medical Center, Guangzhou medical university, \\ ${ }^{3}$ Sun Yet-Sen University Cancer Center \\ ${ }^{4}$ Sun Yet-Sen University
}

September 21, 2020

\begin{abstract}
Objective: We investigated the influence of methylenetetrahydrofolate reductase (MTHFR) C677T/A1298C polymorphisms on the survival of pediatric non-Hodgkin lymphoma (NHL) cases in south China treated with the modified Berlin-FrankfurtMünster 95 protocol. Methods: We reviewed the medical records of 374 patients newly diagnosed at our center between 2014 and 2020. A subgroup of 158 patients was genotyped based on polymorphisms C677T and A1298C. Results: Overall, there were 283 male $(75.7 \%)$ and 91 female patients (24.3\%); the median age was 9 years (range, 1-18 years). The tumor types included Burkitt lymphoma (BL; $\mathrm{n}=180)$, lymphoblastic lymphoma (LBL; $\mathrm{n}=95)$, anaplastic large cell lymphoma $(\mathrm{ALCL} ; \mathrm{n}=64)$, and diffuse large B cell lymphoma (DLBCL; $\mathrm{n}=35)$. At diagnosis, 327 patients (87.4\%) had advanced-stage disease; $159(42.5 \%)$ and 152 patients $(40.6 \%)$ were stratified into the intermediate- and high-risk group, respectively. Seventy (18.7\%) and 36 cases $(9.6 \%)$ had bone marrow and central nervous system (CNS) involvement, respectively. The median follow-up time was 28.5 months (range, 1-76 months); complete remission rate, estimated 5-year event-free survival, and 5-year overall survival rate was $86.1 \%, 74.2 \%$, and $85.7 \%$, respectively. The C677T variant allele was correlated with favorable survival in BL/DLBCL, while ALCL, CNS involvement, advanced stage, and intermediate/high risk were associated with poor survival in NHL. Conclusion: Analysis of the C677T polymorphism could be used for survival prediction and potential risk stratification for further treatment protocols for Chinese pediatric NHL. Further larger studies are needed to verify the clinical value of the C677T polymorphism.
\end{abstract}

\section{INTRODUCTION}

Non-Hodgkin lymphoma (NHL), the fourth most common malignancy across the pediatric age spectrum, is a heterogeneous group of lymphoid malignancies. ${ }^{1,2}$ In children, NHL comprises of four main categories: Burkitt lymphoma (BL), lymphoblastic lymphoma (LBL), diffuse large B-cell lymphoma (DLBCL), and anaplastic large cell lymphoma (ALCL). ${ }^{3,4}$ The current overall survival (OS) rate of pediatric NHL is $>80 \%$ due to dramatic progress in developing risk-adapted curative therapy. ${ }^{1}$

Folate is a vital coenzyme in DNA synthesis. Methylenetetrahydrofolate reductase (MTHFR) plays a key role in intracellular folate metabolism by converting 5,10-methylenetetrahydrofolate (5,10-MTHF), to 5methyltetrahydrofolate (5-MTHF), which is the major circulating form of folate and is essential for protein synthesis and nucleic acid methylation. ${ }^{5-7}$ The two most extensively studied single-nucleotide polymorphisms (SNPs) of MTHFR concerning NHL risk and treatment outcome are the C677T variant (Ala222Val, rs1801133) and the A1298C variant (Glu 429Ala, rs1801131); both dampening enzyme activity by $40-70 \% .5,8,9$ Many studies have investigated the relationships between polymorphisms in the folatemetabolizing MTHFR gene and NHL. Several case-control studies have shown that the correlation between 
C677T/A1298C and NHL risk could be modified by ethnicity. ${ }^{10-15}$ Moreover, MTHFR gene variants play a significant role in the outcome of patients with NHL. Nevertheless, conclusions remain inconsistent. Analysis of polymorphisms in the folate pathway genes might help to reduce chemotherapy toxicity and improve survival by indicating when dose adjustments or alternative treatments are needed. ${ }^{7}$

Recently, there has been growing interest in the effects of the C677T and A1298C on the survival of patients with cancer. Most studies have focused on the survival of pediatric acute lymphoblastic leukemia (ALL), ${ }^{5,16-18}$ osteosarcoma, ${ }^{19}$ adult NHL, ${ }^{7}$ and colorectal cancer (CRC) ${ }^{20-24}$ whereas the relationships between clinical outcome and folate pathway gene variants in pediatric NHL have been poorly investigated. Therefore, the main purpose of the present retrospective study was to evaluate the influence of the C677T and A1298C polymorphisms on the survival of pediatric NHL treated according to the modified NHL-BFM (Berlin-Frankfurt-Münster) 95 protocol.

\section{METHODS}

\subsection{Patients and chemotherapeutic regimens}

We searched the Sun Yat-sen University Cancer Center (SYSUCC) database. The inclusion criteria were: (1) aged [?] 18 years; (2) newly diagnosed as the four main categories of NHL between 2014 and 2020; (3) treated according to the modified BFM95 protocol. At diagnosis, patients were staged using the new International Pediatric NHL Staging System (IPNHLSS). ${ }^{25}$ Based on the clinicopathological features, including stage, surgical outcomes before chemotherapy, LDH (lactate dehydrogenase) levels, pathological subtype and involved organs, patients with BL/DLBCL and ALCL were stratified into four risk groups: very low, low, intermediate, and high; patients with LBL were stratified into low-, intermediate- and high-risk groups according to their stage, genotype, immunophenotyped, and response to induction therapy. This study was approved by the SYSUCC Institutional Review Board and the Research Ethics Committee (IRB: B2020-160-01).

\subsection{Genotypic polymorphism}

MTHFR genetic variations were detected by PCR following Sanger sequencing. The PCR was performed as follows: $94 \operatorname{degC}$ for $5 \mathrm{~min}$, followed by $94 \operatorname{degC}$ for $30 \mathrm{~s}, 58 \operatorname{degC}$ for $30 \mathrm{~s}$, and $72 \operatorname{degC}$ for $30 \mathrm{~s}$ for 32 cycles, and a final extension at $72 \mathrm{degC}$ for $10 \mathrm{~min}$. MTHFR c.677C $>\mathrm{T}$ and c.1298 A $>\mathrm{C}$ were $246 \mathrm{bp}$ and $256 \mathrm{bp}$, respectively. The sequences of the primers used were as follows: c. $677 \mathrm{C}>\mathrm{T}$, forward: 5'TGCCCAGTCCCTGTGGTCTC-3', reverse: 5'-GGCAAGTGATGCCCATGTCG-3'; c.1298A>C, forward: 5'-TTTGGGGAGCTGAAGGACTA-3', reverse: 5'-ACAGGATGGGGAAGTCACAG-3'. The genotypes are presented in Fig. S1 .

\subsection{Follow-up}

Progression (PD) before achieving complete remission (CR), relapse after CR, secondary cancer or Langenhans cell histiocytosis (LCH), and death due to any cause were evaluated as events. The criterion of PD, $\mathrm{CR}$ and stable disease (SD) was in consistence with the International Pediatric NHL Response Criteria. ${ }^{26}$ OS time was calculated from the time of initial diagnosis to death; event-free survival (EFS) time was from the time of initial diagnosis to the first event. Patients who had not died or experienced other events at final analysis were censored at the last follow-up in May 2020.

\subsection{Statistical analyses}

Statistical analyses were performed with IBM SPSS Statistics 25.0 software (SPSS Inc. Headquarters, Chicago, IL, USA). Deviations from the Hardy-Weinberg (H-W) equilibrium of the MTHFR C677T/A1298C genotypes and the associations between categorical variables were tested using the chi-square test. EFS and OS were analyzed according to the Kaplan-Meier (K-M) method using the patient follow-up; differences were compared using the log-rank (Mantel Cox) test. The K-M survival curves were plotted using GraphPad Prism 8.0 (GraphPad Software, San Diego, CA, USA). $P$-values $<0.05$ were considered significant. All data in our study have been recorded at SYSUCC for future reference (RDDA2020001559, 
https://www.researchdata.org.cn).

\section{RESULTS}

\subsection{Patient characteristics}

The clinical characteristics of the investigated group are presented inTABLE 1 . We reviewed a total of 374 pediatric patients with the four NHL subtypes; the median age was 9 years (range, 1-18 years). There were 283 male $(75.7 \%)$ and 91 female patients $(24.3 \%)$. The majority of patients were diagnosed as BL $(n=180 ;$ $48.1 \%)$ and LBL $(n=95 ; 25.4 \%)$; the remaining patients were diagnosed as ALCL $(n=64 ; 17.1 \%)$ and DLBCL $(n=35 ; 9.4 \%)$. There were 327 patients $(87.4 \%)$ at an advanced stage (stage III/IV) at diagnosis; $159(42.5 \%)$ and 152 patients $(40.6 \%)$ were stratified into the intermediate- and high-risk group, respectively. At diagnosis, the incidence rate of bone marrow (BM) and central nervous system (CNS) involvement was 18.7\% (70 cases) and 9.6\% (36 cases), respectively. After BFM 95 chemotherapy, 322 (86.1\%) and 9 patients (2.4\%) achieved CR and SD, respectively. Twenty-one patients (5.6\%) died. Treatment response evaluation was not available in 14 patients $(3.7 \%)$ due to discontinuation of therapy. Of these, eight (2.1\%) were still under treatment at the time of the last follow-up. The median follow-up time was 28.5 months (range, 1-76 months) for all patients. The estimated 5-year EFS and OS were $74.2 \%$ and $85.7 \%$, respectively. In total, events were recorded for 82 patients $(21.9 \%)$, including $\mathrm{PD}(n=29 ; 7.6 \%)$, relapse $(n=31 ; 8.3 \%)$, secondary malignancy or LCH $(n=4 ; 1.1 \%)$, and death $(n=42 ; 11.2 \%)$. Some patients had $>1$ type of event. Among the four cases with secondary malignancy or $\mathrm{LCH}$, one female patient with $\mathrm{BL}$ and one male patient with $\mathrm{B}$ cell-LBL (B-LBL) (CNS-positive) were diagnosed as secondary acute myeloid leukemia (AML-M5) 2 months and 36 months after achieving CR, respectively. They both then died of cancer. One male patient with DLBCL was diagnosed as B cell ALL (B-ALL) (Ph+), and another male patient with T cell LBL (T-LBL) was diagnosed as LCH 15 months and 26 months after achieving CR, respectively. They were both alive and were under chemotherapy at the last follow-up date.

\subsection{Correlation between MTHFR C677T/A1298C and clinicopathologic characteristics of pe- diatric patients with NHL}

Among the whole group, 158 patients (42.2\%) were genotyped with relation to polymorphisms C677T and A1298C. However, MTHFR A1298C sequencing failed in four patients. No significant differences were found in the clinicopathological features between the two subgroups with or without MTHFR genotyping (TABLE 1). Both the C677T and A1298T alleles exhibited the H-W equilibrium in the studied population $\left(677 \mathrm{C}>\mathrm{T}\right.$ variant: $\chi^{2}=0.3326, P=0.5641 ; 1298 \mathrm{~A}>\mathrm{C}$ variant: $\left.\chi^{2}=0.1010, P=0.7506\right)$. Over half of the patients $(83 / 158 ; 52.5 \%)$ carried the C677T wild-type genotype (CC), while $61 / 158(38.6 \%$ ) and 14/158 patients $(8.9 \%)$ carried the heterozygous (CT) and homozygous (TT) genotype, respectively. For the A1298C polymorphism, wild-type (AA), heterozygous (CT) and homozygous (TT) genotypes were observed in $96 / 154(62.3 \%), 52 / 154(33.8 \%)$ and $6 / 154$ patients $(3.9 \%)$, respectively. Interestingly, all patients with $677 \mathrm{TT}(n=14)$ exhibited the wild-type 1298AA genotype. TABLE 2 shows the correlation between the $\mathrm{C} 677 \mathrm{~T} / \mathrm{A} 1298 \mathrm{C}$ polymorphisms and the clinicopathologic characteristics of pediatric patients with NHL. C677T showed a correlation with the incidence of all events $(P=0.042)$. A1298C was found to be significantly associated with NHL subtype $(P=0.045)$.

\subsection{Influence of MTHFR C677T/A1298C on the survival of pediatric NHL}

The median follow-up time was 37 months (range, $1-73$ months) for the subgroup of genotyped patients $(n=$ 158). K-M analysis comparing EFS for the C677T genotypes showed significant differences $(P=0.048 ;$ Fig. 1A.). The estimated 5-year EFS rate for patients with the CC, CT, and TT genotype was $68.0 \%, 87.7 \%$, and $78.6 \%$, respectively. Pairwise comparisons suggested that there was a significant difference only between the $\mathrm{CC}$ and CT genotypes $(P=0.013)$. When the whole subgroup of NHL cases with C677T sequencing was stratified into wild-type and mutant (677T carrier) groups, K-M analysis showed that $677 \mathrm{~T}$ carriers had a better estimated 5-year EFS rate than those with the wild-type genotype (85.9\% vs. $68.0 \% ; P=0.020 ;$ Fig. 1B.). Although no significant difference in OS was found between patients with C677T genotypes $(P=0.495$ or $P=0.312$; Figs. $1 \mathrm{C}$ and $1 \mathrm{D}$ ), similar to EFS, 677 T carriers also had a slightly better 5 -year OS rate than 
those with the wild-type genotype (89.8\% vs. $82.4 \% ; P=0.312$; Fig. 1D). The A1298C polymorphism did not influence the survival of pediatric patients with NHL (Fig. S2A-D). Patients with different genotypes had similar 5-year EFS and OS rate.

Considering that BL and DLBCL are mature B cell NHL, which have the same criteria for risk stratification and treatment regimens but which differ from LBL and ALCL, the NHL subtype might be a confounding factor in analyzing the influence of MTHFR on the whole group of NHL. Accordingly, we performed K-M survival analysis in three subtype groups: BL/DLBCL $(n=81)$, LBL $(n=45)$, and ALCL $(n=32)$. The results indicate that patients with BL/DLBCL carrying the heterozygous C677T genotype had the highest rates for 5-year EFS (97.1\%, Fig. 2A) and 5-year OS (100\%, Fig. 2C) and that T carriers were associated with better EFS and OS rates (Figs. 2B and 2D). However, no significant difference was found in EFS and OS among the A1298C genotypes (Fig. S3). Both the C677T and A1298C polymorphisms did not have any impact on the EFS and OS in patients with LBL and ALCL.

\subsection{Other prognostic factors for the survival of pediatric NHL}

$\mathrm{K}-\mathrm{M}$ analysis was also performed to investigate the impact of other clinicopathological features on the survival of pediatric NHL. NHL subtype, CNS involvement, stage, and risk were significantly associated with EFS or OS. Despite there being no significant difference in 5-year OS rate among the four NHL subtypes (BL, 85.9\%; DLBCL, 96.4\%; LBL 83.5\%; ALCL, $84.6 \% ; P=0.404$;Fig. 3B), the 5 -year EFS rate $(P=0.013$; Fig. 3A) in ALCL (49.0\%) was remarkably lower than that in BL $(79.0 \% ; P=0.021)$, DLBCL $(87.4 \% ; P=0.005)$, and LBL $(76.7 \% ; P=0.006)$. Patients with CNS involvement at diagnosis had a similar 5 -year EFS rate ( $74.7 \%$ vs. $69.0 \%$; $P=0.115$; Fig. $3 \mathrm{C})$, but had a significantly lower 5 -year OS rate $(68.5 \%$ vs. $87.6 \% ; P=$ 0.004; Fig. 3D) than those without. Significant differences were found for both the 5-year EFS rate $(89.0 \%$ vs. $72.2 \% ; P=0.011 ;$ Fig. $4 \mathrm{~A})$ and $\mathrm{OS}$ rate $(100 \%$ vs. $83.7 \% ; P=0.013 ;$ Fig. $4 \mathrm{~B})$ between patients with early (stage I/II) and advanced (stage III/IV) disease. Patients with early-stage disease had better survival than those with advanced -stage disease. Similarly, the very low/low-risk group had significantly better survival than the intermediate/high-risk group: the 5 -year EFS rate was $91.3 \%$ and $70.8 \%(P=0.002$; Fig. $4 \mathrm{C})$, respectively, and the 5-year OS rate was $98.3 \%$ and $83.0 \%(P=0.009$; Fig. $4 \mathrm{D})$, respectively.

\section{DISCUSSION}

We enrolled a homogenous group of 374 children diagnosed with the four main NHL subtypes and who were treated with modified BFM 95 regimens over the past 6 years in south China. Compared to many studies, our sample number was not small for survival analysis. The sex ratio was approximately 3:1, with more males than females. BL and LBL were observed more frequently than DLBCL and ALCL. At diagnosis, the dominant majority of patients had advanced-stage disease and were stratified into intermediateor high-risk group. The incidence rate of BM involvement was about twice that of CNS involvement. Generally, others have reported similar clinical characteristics of pediatric NHL in both white and Asian population. ${ }^{1,27-31}$ Overall, the patients responded well to the chemotherapy regimens at our center. The rates of CR (86.1\%), 5-year EFS $(74.2 \%)$ and OS $(85.7 \%)$ rate in the whole group were comparable to that of different working groups. ${ }^{1,32,33}$ Although less than half of the patients in the present study were genotyped for the C677T/A1298C polymorphism, the subgroup of 158 genotyped patients could be considered as a representative sample of the whole group of pediatric NHL, as no significant difference was found in any clinicopathological characteristics between the two subgroups with or without MTHFR genotyping (TABLE 1). In this analysis, both $\mathrm{C} 677 \mathrm{~T}$ and $\mathrm{A} 1298 \mathrm{C}$ alleles were in $\mathrm{H}-\mathrm{W}$ equilibrium. The mutation rate of $\mathrm{C} 677 \mathrm{~T}$ was slightly greater than that of A1298C (47.5\% vs. 37.7\%). C677T genotype frequencies were similar to that of other reports in Asian pediatric patients with NHL. ${ }^{34,}{ }^{35}$ Data on A1298C are limited in Asia. However, some studies on North American and European children with NHL reported a higher mutation rate of C677T and A1298C. ${ }^{27}, 36$ Therefore, we confirm the high regional and geo-graphic variability of MTHFR polymorphism prevalence in previous publications. ${ }^{8}$ The distribution of A1298C genotypes was significantly different among the NHL subtypes (TABLE 2,P $=0.045$ ); patients with DLBCL had the highest mutation rate $(58.3 \%)$, while patients with LBL had the lowest rate $(28.9 \%)$. 
The influence of MTHFR C677T/A1298C on the survival of patients with cancer (non-hematologic and hematologic malignancy) has attracted great attention from researchers. Regarding adult solid cancers, available evidence on CRC is conflicting; different studies have reported that both C677T and A1298C could be a positive or negative prognostic factor or have no effects on survival of patients with CRC. ${ }^{20-24}$ No significant differences in the OS were found among the wild-type $677 \mathrm{CC}$ and variant $\mathrm{CT} / \mathrm{TT}$ genotypes in patients with glioma. ${ }^{37}$ Martha et al. reported that both the C677T and A1298C polymorphisms were not significantly associated with risk of death, but that the $677 \mathrm{~T}$ variant allele may adversely affect long-term survival in advanced breast cancer. ${ }^{38}$ Lambrecht et al. found that the homozygous 677TT genotype was associated with a higher relapse rate but was not predictive for OS in pediatric osteosarcoma. ${ }^{39}$ Similarly, Xie et al. suggested that genetic polymorphism of MTHFR C677T in the MTX metabolic pathway had no obvious effect on progression-free survival and tumor necrosis rate in sarcoma (age range, 5-52 years). ${ }^{40}$ For hematological malignancy, early studies on childhood ALL have reported controversial results. ${ }^{16-18}$ However, a recent review concluded that there was no association or opposite effects in the majority of published data, and that these two polymorphisms are not suitable predictors of outcome in pediatric ALL. ${ }^{5}$ However, the MTHFR 677TT homozygous genotype has been associated with shorter survival in patients with myelodysplastic syndromes ${ }^{41}$.

Only a few studies have investigated the influence of the C677T and A1298C polymorphisms on the survival of patients with NHL, with controversial results, and there is a particular lack of evidence on A1298C. In an Italian retrospective study with a total of 110 adult patients with NHL treated according to CHOP (cyclophosphamide, doxorubicin hydrochloride, vincristine sulfate, prednisone) or MACOP-B (methotrexate, doxorubicin, cyclophosphamide, vincristine, prednisone, bleomycin) protocol, survival analysis (log-ranks) implied that $677 \mathrm{~T}$ carriers had a significant reduced probability of 5-year EFS in the whole group and in the MACOP-B-treated group $(n=68)$. The difference was more obvious when $1298 \mathrm{CC}$ cases were excluded from the reference group. However, no significant correlations were identified in the CHOP- treated $\operatorname{group}(n$ $=42) .{ }^{7}$ D'Angelo et al. also found that $677 \mathrm{~T}$ carriers could have increased risk of relapse and reduced disease-free survival compared with wild-type patients in Caucasian and European children or adolescent NHL treated with LNH-97 and EURO LB-02 multiagent chemotherapy protocols developed by the AIEOP (Associazione Italiana di Ematologia e Oncologia Pediatrica). ${ }^{27}$ The multicenter NHL-BFM95 trial, involving centers in Austria, Germany, and Switzerland $(n=484)$, determined that the MTHFR $677 \mathrm{C}>\mathrm{T}$ polymorphism was not associated with clinical characteristics (sex, age, tumor stage) and did not appear to influence EFS in pediatric patients with NHL. ${ }^{36}$

However, in our study, the C677T polymorphism was associated with all-events incidence. Patients carrying the wild-type were more likely to experience events. Moreover, K-M survival analysis revealed that $677 \mathrm{~T}$ allele could significantly increase the probability of 5-year EFS and was associated with a non-significant increased probability of 5 -year OS. Therefore, the $677 \mathrm{~T}$ variant allele might have played a protective role in the survival of childhood NHL in the present analysis. The influence of $\mathrm{T}$ variant allele on survival was more obvious when only BL/DLBCL were included in K-M analysis. As no significant difference was found when only LBL or ALCL were included, we assumed that the significant differences in the whole group of NHL resulted from the large proportion of BL/DLBCL cases $(n=81,51.3 \%)$. Furthermore, we speculated that cancer type, age, treatment regimens, and particularly ethnicity might account in part for the discrepant results. A large meta-analysis concluded that the $\mathrm{C} 677 \mathrm{~T}$ polymorphism showed a statistically significantly increased risk for whites, but a decreased risk for Asians ${ }^{6}$. Therefore, it is also possible that the C677T polymorphism has an opposite influence on the survival of European and Chinese pediatric NHL even when patients are treated using the same chemotherapy protocol. The reduced MTHFR activity due to the $677 \mathrm{~T}$ variant allele might have positive effects on survival of pediatric NHL in the Chinese population.

Interestingly, the A1298C polymorphism has been associated with increased NHL risk for in Asians. ${ }^{6}$ In the present study, except for NHL subtype, no significant associations of A1298C in clinical characteristics (age, sex, stage, risk, BM/CNS involvement) and outcome (EFS and OS) could be detected. However, a fact that should not be neglected is that MTHFR enzymatic activity is affected by the A1298C polymorphism to a lesser extent than by $\mathrm{C} 677 \mathrm{~T}$, and the remaining activity in the 1298CC homozygous 
genotype still represents $60 \%$ of the normal status despite of the substitution of glutamate for alanine in the amino acid sequence. ${ }^{5,9,10}$ Furthermore, due to partial linkage disequilibrium, it is possible, but indeed very rare, for $677 \mathrm{~T}$ and $1298 \mathrm{C}$ variant alleles in cis to coexist, supporting the hypothesis that triple mutations $(677 \mathrm{TT} / 1298 \mathrm{AC}$ or $677 \mathrm{CT} / 1298 \mathrm{CC})$ or double homozygous conditions $(677 \mathrm{TT} / 1298 \mathrm{CC})$ are probably de novo recombinant events. ${ }^{42}$ In the present study, virtually all patients with $677 \mathrm{TT}(n=14)$ had the wild-type 1298AA genotype. For these reasons, it was difficult to observe a clear allele-dosage effect for the variant $1298 \mathrm{C}$ being better accounted for in homozygous conditions. This could partly explain the lack of significant associations between the A1298C polymorphism and survival in our study, base on the difficulty in determining the effects of 1298 variant itself on survival.

Our results indicated that patients with ALCL had a significantly higher probability of experiencing events than patients with the other three subtypes, while patients with DLBCL had a relatively superior survival. Overall, patients with ALCL have inferior EFS compared to the other three types. ${ }^{1,30,43}$ Besides, we found that age, sex, and BM involvement did not affect survival, but CNS involvement, advanced stage, and intermediate/high risk predicted inferior survival in pediatric NHL. Similarly, a report from BFM Group study NHL-BFM95 ( $n=505$; age range, 1.4-19.7 years) revealed that CNS-positive patients had remarkably lower 3-year EFS compared to the whole group (69\% vs. $89 \%$ ), and that stage and risk were adversely associated with 3-year EFS. ${ }^{31}$ Besides, a large multicenter retrospective study of 749 pediatric patients with NHL in east Asia suggested that BM involvement does not affect treatment outcome, while CNS involvement and advanced stage ([?] stage III) were associated with poor outcomes $(P<0.05) .{ }^{30}$ More recently, Choeyprasert et al. identified advanced disease stage as a significant poor prognostic factor for 5 -year EFS of pediatric NHL in Thailand. ${ }^{29}$

We are aware of the limitations of our study, given the retrospective nature and relatively small sample size enrolled for analyzing the influence of MTHFR on the survival of pediatric NHL. Moreover, not all newly diagnosed pediatric NHL at our center during the study period were included for analysis, as a small group of them did not receive modified BFM 95 regimens, but were alternatively treated with an adult protocol such as the R(rituximab)-CHOP regimen.

In conclusion, we found that the MTHFR C677T polymorphism and some clinicopathological characteristics including, NHL subtype, CNS involvement, stage, and risk at diagnosis, are significantly related to the survival of pediatric patients with NHL treated with modified BFM 95 regimens. The $677 \mathrm{~T}$ allele could increase the probability of 5-year EFS and 5-year OS. No evidence showed that the MTHFR A1298C polymorphism influenced the EFS or OS rate in pediatric patients with NHL. Further larger studies are encouraged to verify these results properly.

\section{Conflict of Interest statement}

The authors declare that they have no conflict of interest.

\section{Acknowledgements}

None

\section{References}

1. Minard-Colin V, Brugieres L, Reiter A, et al. Non-Hodgkin Lymphoma in Children and Adolescents: Progress Through Effective Collaboration, Current Knowledge, and Challenges Ahead. J Clin Oncol . Sep 20 2015;33(27):2963-74. doi:10.1200/JCO.2014.59.5827

2. Gu X, Zheng R, Xia C, et al. Interactions between life expectancy and the incidence and mortality rates of cancer in China: a population-based cluster analysis. Cancer Commun (Lond) . Jul 3 2018;38(1):44. doi:10.1186/s40880-018-0308-x

3. Marginean CO, Melit LE, Horvath E, Gozar H, Chincesan MI. Non-Hodgkin lymphoma, diagnostic, and prognostic particularities in children - a series of case reports and a review of the literature (CARE compliant).Medicine (Baltimore) . Feb 2018;97(8):e9802. doi:10.1097/MD.0000000000009802 
4. Sandlund JT, Martin MG. Non-Hodgkin lymphoma across the pediatric and adolescent and young adult age spectrum. Hematology Am Soc Hematol Educ Program . Dec 2 2016;2016(1):589-597. doi:10.1182/asheducation-2016.1.589

5. Umerez M, Gutierrez-Camino A, Munoz-Maldonado C, Martin-Guerrero I, Garcia-Orad A. MTHFR polymorphisms in childhood acute lymphoblastic leukemia: influence on methotrexate therapy. Pharmgenomics Pers Med . 2017;10:69-78. doi:10.2147/PGPM.S107047

6. He J, Liao XY, Zhu JH, et al. Association of MTHFR C677T and A1298C polymorphisms with non-Hodgkin lymphoma susceptibility: evidence from a meta-analysis. Sci Rep . Aug 22 2014;4:6159. doi:10.1038/srep06159

7. Gemmati D, Ongaro A, Tognazzo S, et al. Methylenetetrahydrofolate reductase C677T and A1298C gene variants in adult non-Hodgkin's lymphoma patients: association with toxicity and survival. Haematologica . Apr 2007;92(4):478-85. doi:10.3324/haematol.10587

8. De Mattia E, Toffoli G. C677T and A1298C MTHFR polymorphisms, a challenge for antifolate and fluoropyrimidine-based therapy personalisation. Eur J Cancer. May 2009;45(8):1333-51. doi:10.1016/j.ejca.2008.12.004

9. Weisberg I, Tran P, Christensen B, Sibani S, Rozen R. A second genetic polymorphism in methylenetetrahydrofolate reductase (MTHFR) associated with decreased enzyme activity. Mol Genet Metab . Jul 1998;64(3):169-72. doi:10.1006/mgme.1998.2714

10. Mashhadi MA, Miri-Moghaddam E, Arbabi N, et al. C677T and A1298C polymorphisms of methylene tetrahydrofolate reductase in non-Hodgkin lymphoma: southeast Iran. Tumori . Aug 2018;104(4):280-284. doi: $10.5301 / \mathrm{tj} .5000634$

11. Skibola CF, Forrest MS, Coppede F, et al. Polymorphisms and haplotypes in folate-metabolizing genes and risk of non-Hodgkin lymphoma. Blood . Oct 1 2004;104(7):2155-62. doi:10.1182/blood-2004-02-0557

12. Suthandiram S, Gan GG, Zain SM, et al. Polymorphisms in methylenetetrahydrofolate reductase gene and risk of non-Hodgkin lymphoma in a multi-ethnic population. J Hum Genet. May 2014;59(5):280-7. doi:10.1038/jhg.2014.19

13. Xie SZ, Liu ZZ, Yu JH, et al. Association between the MTHFR C677T polymorphism and risk of cancer: evidence from 446 case-control studies. Tumour Biol . Nov 2015;36(11):8953-72. doi:10.1007/s13277015-3648-z

14. Kim HN, Lee IK, Kim YK, et al. Association between folate-metabolizing pathway polymorphism and non-Hodgkin lymphoma.Br J Haematol . Feb 2008;140(3):287-94. doi:10.1111/j.1365-2141.2007.06893.x

15. Lee KM, Lan Q, Kricker A, et al. One-carbon metabolism gene polymorphisms and risk of non-Hodgkin lymphoma in Australia. Hum Genet . Dec 2007;122(5):525-33. doi:10.1007/s00439-007-0431-2

16. Ojha RP, Gurney JG. Methylenetetrahydrofolate reductase C677T and overall survival in pediatric acute lymphoblastic leukemia: a systematic review. Leuk Lymphoma . Jan 2014;55(1):67-73. doi:10.3109/10428194.2013.792336

17. de Deus DM, de Lima EL, Seabra Silva RM, Leite EP, Cartaxo Muniz MT. Influence of Methylenetetrahydrofolate Reductase C677T, A1298C, and G80A Polymorphisms on the Survival of Pediatric Patients with Acute Lymphoblastic Leukemia. Leuk Res Treatment . 2012;2012:292043. doi:10.1155/2012/292043

18. Karas Kuzelicki N, Milek M, Jazbec J, Mlinaric-Rascan I. 5,10-Methylenetetrahydrofolate reductase (MTHFR) low activity genotypes reduce the risk of relapse-related acute lymphoblastic leukemia (ALL).Leuk Res . Oct 2009;33(10):1344-8. doi:10.1016/j.leukres.2008.12.011

19. Lambrecht L, Sleurs C, Labarque V, Dhooge C, Uyttebroeck A. The role of the MTHFR C677T polymorphism in methotrexate-induced toxicity in pediatric osteosarcoma patients. Pharmacogenomics . 
2017;18(8):787-795.

20. Zhu L, Wang F, Hu F, et al. Association between MTHFR polymorphisms and overall survival of colorectal cancer patients in Northeast China.Med Oncol . Mar 2013;30(1):467. doi:10.1007/s12032-0130467-1

21. Yeh CC, Lai CY, Chang SN, et al. Polymorphisms of MTHFR C677T and A1298C associated with survival in patients with colorectal cancer treated with 5-fluorouracil-based chemotherapy. Int J Clin Oncol . Jun 2017;22(3):484-493. doi:10.1007/s10147-016-1080-z

22. Liu D, Li X, Li X, et al. CDA and MTHFR polymorphisms are associated with clinical outcomes in gastroenteric cancer patients treated with capecitabine-based chemotherapy. Cancer Chemother Pharmacol . May 2019;83(5):939-949. doi:10.1007/s00280-019-03809-2

23. Ose J, Botma A, Balavarca Y, et al. Pathway analysis of genetic variants in folate-mediated one-carbon metabolism-related genes and survival in a prospectively followed cohort of colorectal cancer patients. Cancer Med . May 29 2018;doi:10.1002/cam4.1407

24. Osian G, Procopciuc L, Vlad L, Iancu C, Mocan T, Mocan L. C677T and A1298C mutations in the MTHFR gene and survival in colorectal cancer.J Gastrointestin Liver Dis . Dec 2009;18(4):455-60.

25. Rosolen A, Perkins SL, Pinkerton CR, et al. Revised International Pediatric Non-Hodgkin Lymphoma Staging System. J Clin Oncol . Jun 20 2015;33(18):2112-8. doi:10.1200/JCO.2014.59.7203

26. Sandlund JT, Guillerman RP, Perkins SL, et al. International Pediatric Non-Hodgkin Lymphoma Response Criteria. J Clin Oncol . Jun 20 2015;33(18):2106-11. doi:10.1200/JCO.2014.59.0745

27. D'Angelo V, Ramaglia M, Iannotta A, et al. Influence of methylenetetrahydrofolate reductase gene polymorphisms on the outcome of pediatric patients with non-Hodgkin lymphoma treated with high-dose methotrexate. Leuk Lymphoma . Dec 2013;54(12):2639-44. doi:10.3109/10428194.2013.784758

28. Gross TG, Termuhlen AM. Pediatric non-Hodgkin's lymphoma. Curr Oncol Rep . Nov 2007;9(6):459-65. doi:10.1007/s11912-007-0064-6

29. Choeyprasert W, Anurathapan U, Pakakasama S, et al. Pediatric non-Hodgkin lymphoma: Characteristics, stratification, and treatment at a single institute in Thailand. Pediatr Int . Jan 2019;61(1):49-57. doi:10.1111/ped.13739

30. Suh JK, Gao YJ, Tang JY, et al. Clinical Characteristics and Treatment Outcomes of Pediatric Patients with Non-Hodgkin Lymphoma in East Asia. Cancer Res Treat . Apr 2020;52(2):359-368. doi:10.4143/crt.2019.219

31. Woessmann W, Seidemann K, Mann G, et al. The impact of the methotrexate administration schedule and dose in the treatment of children and adolescents with B-cell neoplasms: a report of the BFM Group Study NHL-BFM95. Blood . Feb 1 2005;105(3):948-58. doi:10.1182/blood-2004-03-0973

32. Muller J, Csoka M, Jakab Z, Ponyi A, Erlaky H, Kovacs G. Treatment of pediatric non-Hodgkin lymphoma in Hungary: 15 years experience with NHL-BFM 90 and 95 protocols. Pediatr Blood Cancer. Mar 2008;50(3):633-5. doi:10.1002/pbc.21144

33. Burkhardt B, Oschlies I, Klapper W, et al. Non-Hodgkin's lymphoma in adolescents: experiences in 378 adolescent NHL patients treated according to pediatric NHL-BFM protocols. Leukemia . Jan 2011;25(1):15360. doi:10.1038/leu.2010.245

34. Shimasaki N, Mori T, Samejima H, et al. Effects of methylenetetrahydrofolate reductase and reduced folate carrier 1 polymorphisms on high-dose methotrexate-induced toxicities in children with acute lymphoblastic leukemia or lymphoma. J Pediatr Hematol Oncol . Feb 2006;28(2):64-8. doi:10.1097/01.mph.0000198269.61948.90 
35. Shimasaki N, Mori T, Torii C, et al. Influence of MTHFR and RFC1 polymorphisms on toxicities during maintenance chemotherapy for childhood acute lymphoblastic leukemia or lymphoma. J Pediatr Hematol Oncol . May 2008;30(5):347-52. doi:10.1097/MPH.0b013e318165b25d

36. Seidemann K, Book M, Zimmermann M, et al. MTHFR $677(\mathrm{C}->\mathrm{T})$ polymorphism is not relevant for prognosis or therapy-associated toxicity in pediatric NHL: results from 484 patients of multicenter trial NHL-BFM 95. Ann Hematol . May 2006;85(5):291-300. doi:10.1007/s00277-005-0072-2

37. Pandith AA, Qasim I, Zahoor W, Shah P, Bhat AR. ACE I/D sequence variants but not MTHFR C677T, is strongly linked to malignant glioma risk and its variant DD genotype may act as a promising predictive biomarker for overall survival of glioma patients. Gene. Jan 10 2018;639:62-68. doi:10.1016/j.gene.2017.10.013

38. Shrubsole MJ, Shu XO, Ruan ZX, et al. MTHFR genotypes and breast cancer survival after surgery and chemotherapy: a report from the Shanghai Breast Cancer Study. Breast Cancer Res Treat . May 2005;91(1):73-9. doi:10.1007/s10549-004-7265-6

39. Lambrecht L, Sleurs C, Labarque V, et al. The role of the MTHFR C677T polymorphism in methotrexate-induced toxicity in pediatric osteosarcoma patients. Pharmacogenomics . Jun 2017;18(8):787795. doi:10.2217/pgs-2017-0013

40. Xie L, Guo W, Yang Y, Ji T, Xu J. More severe toxicity of genetic polymorphisms on MTHFR activity in osteosarcoma patients treated with high-dose methotrexate. Oncotarget . Feb 20 2018;9(14):11465-11476. doi:10.18632/oncotarget.23222

41. Visani G, Loscocco F, Ruzzo A, et al. MTHFR, TS and XRCC1 genetic variants may affect survival in patients with myelodysplastic syndromes treated with supportive care or azacitidine. Pharmacogenomics $J$ . May 22 2018;18(3):444-449. doi:10.1038/tpj.2017.48

42. Zetterberg H, Rymo L, Coppola A, D'Angelo A, Spandidos DA, Blennow K. Reply to 'MTHFR C677T and A1298C polymorphisms and mutated sequences occurring in cis'. European Journal of Human Genetics . 2002/10/01 2002;10(10):579-582. doi:10.1038/sj.ejhg.5200863

43. Sandlund JT. Non-Hodgkin Lymphoma in Children. Curr Hematol Malig Rep . Sep 2015;10(3):237-243. doi:10.1007/s11899-015-0277-y

\section{Legends}

Figure 1. Kaplan-Meier analysis of event-free survival (EFS) and overall survival (OS) in the subgroup of pediatric NHL with genotype of the C677T polymorphism $(n=158)$. WT, wild genotype (CC); Mut, mutant genotype $(\mathrm{CT} / \mathrm{TT})$.

A. The 5-year EFS rate for CC, CT, and TT genotype was $68.0 \%, 87.7 \%$, and $78.6 \%$, respectively; CC/CT: $P=0.013 ; \mathrm{CC} / \mathrm{TT}: P=0.633 ; \mathrm{CT} / \mathrm{TT}: P=0.306$;

B. Patients carrying mutant genotype had a significantly higher 5-year EFS rate than those with wild type (85.9\% vs. $68.0 \%)$;

C-D. No significant difference was found in OS among different genotypes.

Figure 2. Kaplan-Meier analysis of event-free survival (EFS) and overall survival (OS) in the subgroup of pediatric BL and DLBCL with genotype of the C677T polymorphism $(n=81)$. WT, wild genotype (CC); Mut, mutant genotype (CT/TT).

A. The 5-year EFS rate for CC, CT, and TT genotype was $70.3 \%, 97.1 \%$, and $85.7 \%$, respectively; CC/CT: $P=0.004 ; \mathrm{CC} / \mathrm{TT}: P=0.511 ; \mathrm{CT} / \mathrm{TT}: P=0.210$;

B. Patients carrying mutant genotype had a significantly higher 5-year EFS rate than those with wild type (95.1\% vs. $70.3 \%)$; 
C. The 5-year OS rate for CC, CT, and TT genotype was $81.9 \%, 100 \%$, and $83.3 \%$, respectively; CC/CT: $P$ $=0.010 ; \mathrm{CC} / \mathrm{TT}: P=0.779 ; \mathrm{CT} / \mathrm{TT}: P=0.021$;

D. Patients carrying mutant genotype had a significantly higher 5 -year OS rate than those with wild type (97.4\% vs. $81.9 \%)$.

Figure 3. Kaplan-Meier analysis of event-free survival (EFS) and overall survival (OS) in the whole group of pediatric NHL stratified by cancer type (A-B) and CNS involvement (C-D) $(n=374)$. CNS, central nervous system.

A. The 5-year EFS rate in ALCL (49.0\%) was significantly lower than that in BL $(79.0 \% ; P=0.021)$, DLBCL $(87.4 \% ; \mathrm{P}=0.005)$, and LBL $(76.7 \% ; P=0.006)$;

B. There was no significant difference in 5-year OS rate among the four NHL subtypes (BL, 85.9\%; LBL 83.5\%; ALCL, $84.6 \%$; DLBCL, 96.4\%);

C. Patients with CNS involvement at diagnosis had a similar 5-year EFS rate with those without (74.7\% vs. $69.0 \%)$

D. Patients with CNS involvement at diagnosis had a significantly lower 5-year OS rate than those without (68.5\% vs. $87.6 \%)$.

Figure 4. Kaplan-Meier analysis of event-free survival (EFS) and overall survival (OS) in the whole group of pediatric NHL stratified by stage (A-B) and risk (C-D) $(n=374)$. Early, stage I \&II; advanced, stage III \& IV.

A. The 5-year EFS rate of patients at advanced stage was significantly lower than that at early stage $(89.0 \%$ vs. $72.2 \%)$;

B. The 5-year OS rate of patients at advanced stage was significantly lower than that at early stage (100\% vs. $83.7 \%$;

C. The 5-year EFS rate of intermediate-/high-risk patients was significantly lower than that of very low/low-risk (91.3\% vs. $70.8 \%)$;

D. The 5-year OS rate of intermediate-/high-risk patients was significantly lower than that of very low-/lowrisk $(98.3 \%$ vs. $83.0 \%)$.

Figure S1. Genetic variations of MTHFR C677T(A-C) and A1298C (D-F) were detected by PCR following Sanger sequencing.

Figure S2. Kaplan-Meier analysis of event-free survival (EFS) and overall survival (OS) in the subgroup of pediatric NHL with genotype of the A1298C polymorphism $(n=154)$. WT, wild genotype (AA); Mut, mutant genotype $(\mathrm{AC} / \mathrm{CC})$. No significant difference was found in EFS and OS among different genotypes.

Figure S3. Kaplan-Meier analysis of event-free survival (EFS) and overall survival (OS) in the subgroup of pediatric BL and DLBCL with genotype of the A1298C polymorphism $(n=78)$. WT, wild genotype (AA); Mut, mutant genotype (AC/CC). No significant difference was found in EFS and OS among different genotypes.

\section{Hosted file}

TABLE 1.docx available at https://authorea.com/users/359973/articles/481717-influence-ofmthfr-c677t-and-a1298c-polymorphisms-on-the-survival-of-pediatric-patients-with-nonhodgkin-lymphoma

\section{Hosted file}

TABLE 2.docx available at https://authorea.com/users/359973/articles/481717-influence-ofmthfr-c677t-and-a1298c-polymorphisms-on-the-survival-of-pediatric-patients-with-non- 
hodgkin-lymphoma
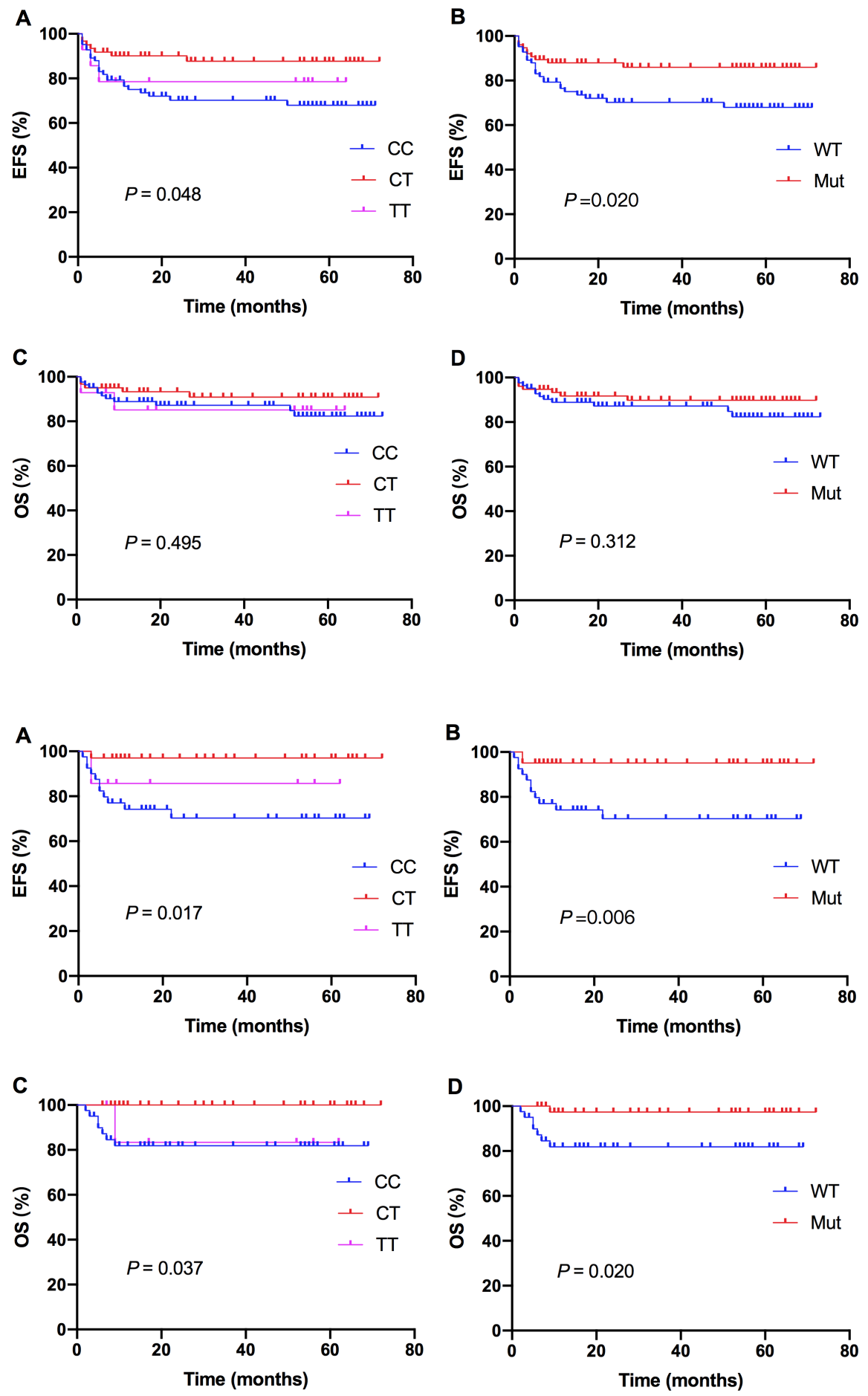
A

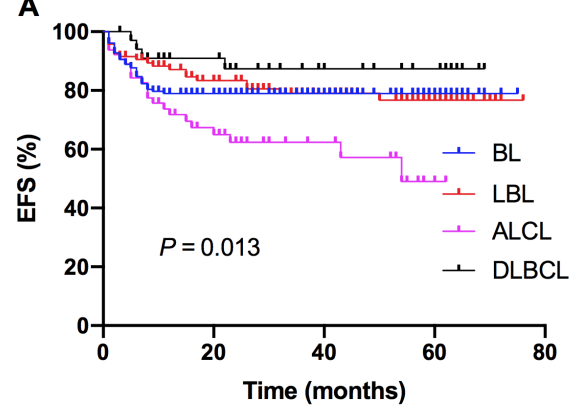

C

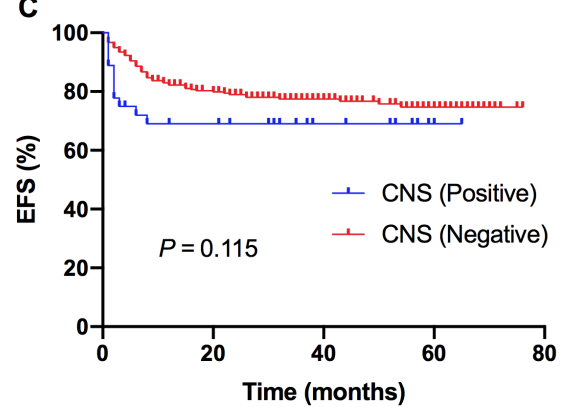

A

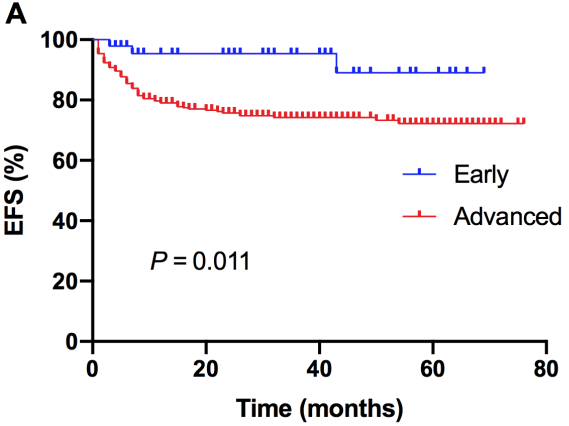

C

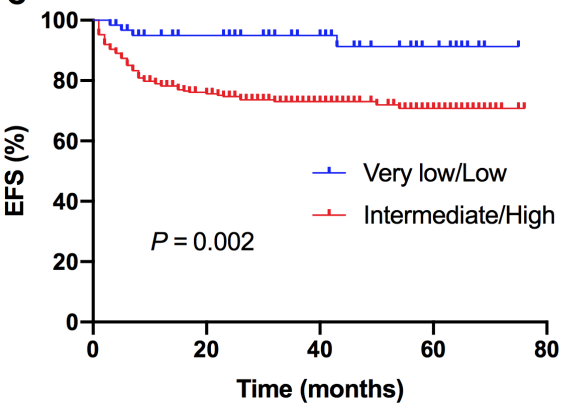

B

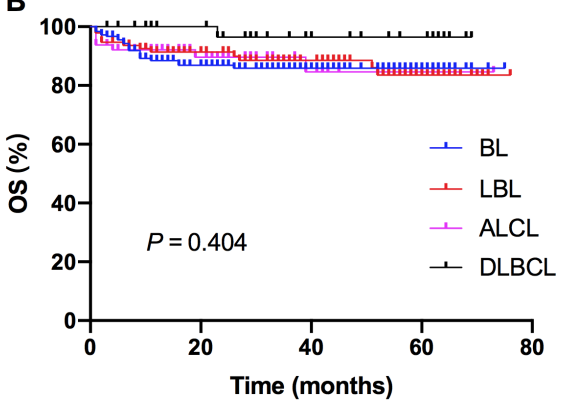

D

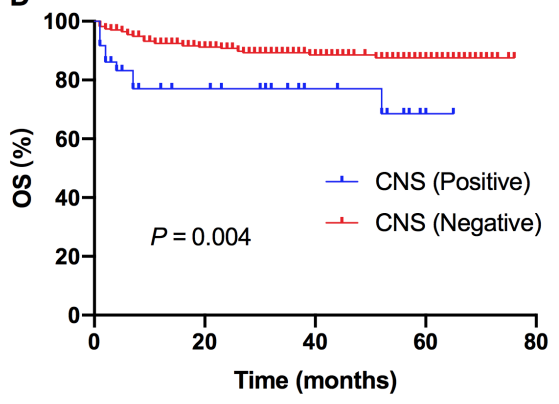

B

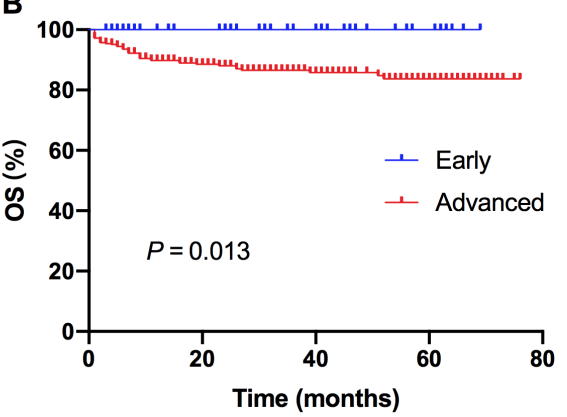

D

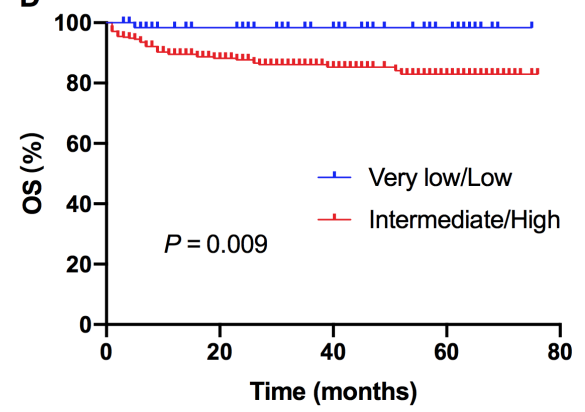

